\title{
Are elevated circulating intercellular adhesion molecule 1 levels more strongly predictive of diabetes than vascular risk? Outcome of a prospective study in the elderly
}

\author{
N. Sattar • H. M. Murray • P. Welsh • G. J. Blauw • \\ B. M. Buckley • A. J. de Craen • I. Ford • \\ N. G. Forouhi • D. J. Freeman • J. W. Jukema • \\ P. W. Macfarlane • M. B. Murphy • C. J. Packard • \\ D. J. Stott • R. G. J. Westendorp • J. Shepherd • \\ for the Prospective Study of Pravastatin in the Elderly \\ at Risk Trial Study Group
}

Received: 15 September 2008 / Accepted: 28 October 2008 / Published online: 22 November 2008

(C) Springer-Verlag 2008

\begin{abstract}
Aims/hypothesis The aim of this prospective study was to determine whether circulating intercellular adhesion molecule (ICAM) 1, as a potential surrogate of 'endothelial activation', is more strongly associated with risk of vascular events than with incident diabetes.
\end{abstract}

N. Sattar $(\bowtie) \cdot$ P. Welsh • D. J. Freeman • P. W. Macfarlane •

C. J. Packard $\cdot$ D. J. Stott $\cdot$ J. Shepherd

Faculty of Medicine, BHF Glasgow Cardiovascular Research

Centre, University of Glasgow,

University Place,

Glasgow G12 8TA, UK

e-mail: nsattar@clinmed.gla.ac.uk

H. M. Murray · I. Ford

Robertson Centre for Biostatistics, University of Glasgow,

Glasgow, UK

G. J. Blauw · A. J. de Craen · R. G. J. Westendorp

Section of Gerontology and Geriatrics,

Leiden University Medical Centre,

Leiden, the Netherlands

B. M. Buckley • M. B. Murphy

Department of Pharmacology and Therapeutics,

Cork University Hospital,

Wilton, Cork, Ireland

N. G. Forouhi

MRC Epidemiology Unit, Institute of Metabolic Science,

Addenbrookes Hospital,

Cambridge, UK

J. W. Jukema

Department of Cardiology, Leiden University Medical Centre,

Leiden, the Netherlands
Methods We related baseline ICAM-1 levels to vascular events (866 CHD and stroke events in 5,685 participants) and incident diabetes (292 in 4,945 without baseline diabetes) in the elderly over 3.2 years of follow-up.

Results ICAM-1 levels correlated positively with triacylglycerol but negatively with LDL- and HDL-cholesterol. ICAM-1 levels were higher in those who developed diabetes $(388.6 \pm 1.42$ vs $369.4 \pm 1.39 \mathrm{ng} / \mathrm{ml}[$ mean $\pm \mathrm{SD}$ ], $p=0.011)$ and remained independently associated with newonset diabetes (HR 1.84, 95\% CI 1.26-2.69, $p=0.0015$ per unit increase in $\log [\mathrm{ICAM}-1]$ after adjusting for classical risk factors and C-reactive protein). By contrast, ICAM-1 levels were not significantly $(p=0.40)$ elevated in those who had an incident vascular event compared with those who remained event-free, and corresponding adjusted risk associations were null (HR $0.98,95 \%$ CI $0.80-1.22, p=$ 0.89 ) in analyses adjusted for other risk factors.

Conclusions/interpretation We show that elevated ICAM-1 levels are associated with risk of incident diabetes in the elderly at risk, despite no association with incident cardiovascular disease risk. We suggest that perturbations in circulating ICAM-1 levels are aligned more towards diabetes risk.

Keywords Adhesion molecules C CVD · Diabetes .

ICAM-1 $\cdot$ Risk factors

$\begin{array}{ll}\text { Abbreviations } \\ \text { CRP } & \text { C-reactive protein } \\ \text { CVD } & \text { cardiovascular disease } \\ \text { ICAM-1 } & \text { intercellular adhesion molecule } 1\end{array}$




\section{Introduction}

Investigators have measured and related soluble forms of cell adhesion molecules to vascular disease in a variety of study types, with intercellular adhesion molecule 1 (ICAM1) the most widely studied. Early prospective studies showed significant associations between baseline circulating ICAM-1 levels and incident vascular events [1-4], but with larger studies and more rigorous adjustment for potential confounders, associations became far less marked [5]. More recently, circulating ICAM-1 levels have been shown to predict diabetes in middle-aged individuals $[6,7]$ but data in the elderly are lacking. We recently suggested that few, if any, risk factors predict both incident diabetes and cardiovascular disease (CVD) events to similar extents [8]. In this paper we present the first simultaneous assessment of the association of any adhesion molecule with risk of incident diabetes and incident CVD events. Our hypothesis was that elevations in ICAM-1 would predict diabetes more strongly than they would predict vascular events. This is an important question, since ICAM-1 is regularly discussed as a vascular-risk surrogate.

\section{Methods}

The methods of the Prospective Study of Pravastatin in the Elderly at Risk trial as an ancillary risk-association study have been reported [9]. Between 1997 and 1999, 5,804 men and women aged 70-82 years were recruited if they had either pre-existing vascular disease or raised risk of such disease because of risk factors. The institutional ethics review boards of all centres approved the protocol, and all participants gave written informed consent. The protocol was consistent with the Declaration of Helsinki. The primary outcome was definite or suspect death from CHD, non-fatal myocardial infarction and fatal or non-fatal stroke. New-onset diabetes was classified by self-reported history, or a fasting blood glucose concentration of $\geq 7.0 \mathrm{mmol} / 1$ or a blood glucose measurement of $\geq 11.1 \mathrm{mmol} / 1$ when fasting status was uncertain (baseline and follow-up glucose measurements were fasting in $>95 \%$ of participants) as detailed before [9]. Participants with known $(n=623)$ and undiagnosed baseline diabetes $(n=$ $137)$ were excluded from analyses. All CVD events were validated by a blinded endpoints committee.

Baseline ICAM-1 was measured by ELISA (R\&D Systems, Abingdon, UK) on previously unthawed prerandomisation plasma samples stored at $-80^{\circ} \mathrm{C}$. The method has an inter-assay $\mathrm{CV}$ of $<7 \%$. Samples were processed blinded to the identity of samples.

The distribution of ICAM-1 was positively skewed and a logarithmic transformation $\left(\log _{\mathrm{e}}\right)$ was used. Relationships between $\log _{\mathrm{e}}[\mathrm{ICAM}-1]$ and baseline characteristics were assessed using Pearson's correlation coefficient for continuous variables and two-sample Student's $t$ tests or ANOVA for categorical variables. The influence of ICAM-1 on the endpoints of interest (diabetes and CVD) was investigated using Cox proportional hazards models, adjusting for randomised treatment and classic and novel risk factors (age, sex, country, LDL- and HDL-cholesterol, triacylglycerol, BP, smoking, BMI, use of antihypertensives, history of vascular disease and C-reactive protein [CRP]), plus glucose for diabetes endpoints only or diabetes for CVD endpoints only. Results are reported as HRs with $95 \%$ CIs for change of 1 unit increases in $\log _{e}[$ ICAM-1] and corresponding $p$ values.

\section{Results}

Baseline analyses in relation to CVD outcomes ICAM-1 levels were available in 5,685 (97.9\%) of the original 5,804 participants. Their baseline characteristics, by endpoint, are shown in Table 1.

Compared with those without follow-up CVD events, those developing a CVD endpoint were slightly older, and more likely to be male and to have a history of diabetes or vascular disease. Total cholesterol and HDL-cholesterol and diastolic BP were lower in those who had a primary event, but fasting glucose after excluding those with diabetes was not higher. Baseline ICAM-1 levels were also not higher in those who had a CVD event. Participants who developed diabetes had a higher fasting glucose, BMI and triacylglycerol and lower HDL-cholesterol, and were more likely to be hypertensive. Baseline ICAM-1 levels were significantly higher among those who developed diabetes.

Correlations of ICAM-1 with other risk variables At baseline, ICAM-1 levels correlated positively but weakly with triacylglycerol (age-, sex-, smoking status-, BMI- and country-adjusted, $r=0.063, p<0.0001)$ and negatively with total cholesterol and HDL- and LDL-cholesterol levels (adjusted $r=-0.035,-0.082$ and -0.031 , respectively, all $p \leq 0.02)$. ICAM-1 also correlated with CRP $(r=0.133, p<$ $0.0001)$ and fasting glucose $(r=0.057, p<0.0001)$. ICAM-1 levels were higher in smokers, and in those with known diabetes or a history of peripheral arterial disease (all $p<0.001)$.

Associations of elevated ICAM-1 with risk of incident vascular events and diabetes There was also no significant interaction in the association of ICAM-1 with CVD events with statin allocation (data not shown). ICAM-1 was not significantly related to risk of either primary CVD endpoint or, taken separately, either coronary events or stroke events 
Table 1 Comparison of baseline characteristics by CVD endpoint (combined endpoint) and by incident diabetes in all those without diabetes at baseline

\begin{tabular}{|c|c|c|c|c|c|c|}
\hline \multirow[t]{2}{*}{ Variable } & \multicolumn{3}{|c|}{ Incident CVD events } & \multicolumn{3}{|l|}{ Incident diabetes } \\
\hline & Event $(n=866)$ & No event $(n=4,819)$ & $p$ value $^{\mathrm{a}}$ & Diabetes $(n=292)$ & No diabetes $(n=4,653)$ & $p$ value $^{\mathrm{a}}$ \\
\hline \multicolumn{7}{|c|}{ Continuous variables, mean \pm SD } \\
\hline Age (years) & $75.8 \pm 3.5$ & $75.3 \pm 3.3$ & $<0.0001$ & $75.4 \pm 3.3$ & $75.4 \pm 3.4$ & 0.94 \\
\hline BMI $\left(\mathrm{kg} / \mathrm{m}^{2}\right)$ & $26.9 \pm 4.1$ & $26.8 \pm 4.2$ & 0.54 & $28.8 \pm 4.5$ & $26.5 \pm 4.1$ & $<0.0001$ \\
\hline Systolic BP (mmHg) & $155.2 \pm 22.9$ & $154.5 \pm 21.6$ & 0.35 & $156.5 \pm 23.7$ & $153.8 \pm 21.5$ & 0.040 \\
\hline Diastolic BP (mmHg) & $82.9 \pm 11.8$ & $83.9 \pm 11.3$ & 0.026 & $84.2 \pm 12.3$ & $83.6 \pm 11.2$ & 0.38 \\
\hline Total cholesterol $(\mathrm{mmol} / \mathrm{l})$ & $5.62 \pm 0.87$ & $5.69 \pm 0.91$ & 0.047 & $5.72 \pm 0.90$ & $5.72 \pm 0.91$ & 0.94 \\
\hline HDL-cholesterol (mmol/l) & $1.24 \pm 0.34$ & $1.29 \pm 0.35$ & $<0.0001$ & $1.21 \pm 0.37$ & $1.30 \pm 0.35$ & $<0.0001$ \\
\hline LDL-cholesterol (mmol/l) & $3.76 \pm 0.76$ & $3.79 \pm 0.80$ & 0.24 & $3.80 \pm 0.76$ & $3.83 \pm 0.80$ & 0.56 \\
\hline Triacylglycerol $(\mathrm{mmol} / \mathrm{l})^{\mathrm{b}}$ & $1.44 \pm 1.53$ & $1.41 \pm 1.51$ & 0.14 & $1.64 \pm 1.56$ & $1.37 \pm 1.50$ & $<0.0001$ \\
\hline $\mathrm{CRP}(\mathrm{mg} / \mathrm{l})^{\mathrm{b}}$ & $3.64 \pm 3.08$ & $3.01 \pm 3.05$ & $<0.0001$ & $3.46 \pm 3.14$ & $3.04 \pm 3.06$ & 0.058 \\
\hline Glucose $(\mathrm{mmol} / \mathrm{l})^{\mathrm{c}}$ & $5.18 \pm 1.02$ & $5.15 \pm 0.82$ & 0.31 & $5.87 \pm 0.76$ & $5.02 \pm 0.60$ & $<0.0001$ \\
\hline ICAM-1 (ng/ml) ${ }^{\mathrm{b}}$ & $374.7 \pm 1.4$ & $370.8 \pm 1.4$ & 0.40 & $388.6 \pm 1.42$ & $369.4 \pm 1.39$ & 0.011 \\
\hline \multicolumn{7}{|l|}{ Categorical variables, $n(\%)$} \\
\hline Men & $494(57.0)$ & $2,252(46.7)$ & $<0.0001$ & $145(49.7)$ & $2,180(46.8)$ & 0.35 \\
\hline \multicolumn{7}{|l|}{ Smoking } \\
\hline Never & $269(31.1)$ & $1,657(34.4)$ & 0.091 & $107(36.6)$ & $1,543(33.2)$ & 0.049 \\
\hline Current smoker & $230(26.6)$ & $1,292(26.8)$ & & $65(22.3)$ & $1,347(29.0)$ & \\
\hline Ex-smoker & $367(42.4)$ & $1,870(38.8)$ & & $120(41.1)$ & $1,763(37.9)$ & \\
\hline \multicolumn{7}{|l|}{ History of } \\
\hline Diabetes & $129(14.9)$ & $477(9.9)$ & $<0.0001$ & - & - & - \\
\hline Hypertension & $521(60.2)$ & $2,994(62.1)$ & 0.27 & $205(70.2)$ & 2,881 (61.9) & 0.0046 \\
\hline Coronary disease & $380(43.9)$ & $1,438(29.8)$ & $<0.0001$ & $98(33.6)$ & $1,511(32.5)$ & 0.70 \\
\hline Peripheral arterial disease & $139(16.1)$ & $497(10.3)$ & $<0.0001$ & $30(10.3)$ & $525(11.3)$ & 0.60 \\
\hline Stroke or TIA & $126(14.5)$ & $512(10.6)$ & 0.0008 & $30(10.3)$ & $544(11.7)$ & 0.46 \\
\hline Any vascular disease & $492(56.8)$ & $2,025(42.0)$ & $<0.0001$ & $123(42.1)$ & $2115(45.4)$ & 0.27 \\
\hline
\end{tabular}

The $p$ values for continuous variables are from two-sample Student's $t$ tests, and those for categorical variables are from $\chi^{2}$ tests. $n$ is the maximum number. All available data were used

${ }^{a}$ Because of the design structure of the trial (recruiting a greater proportion of participants with hypertension and diabetes and smokers and women into the low-risk primary-prevention group) the significance or non-significance of univariate comparisons in this table should be treated with caution

${ }^{\mathrm{b}}$ Values are geometric means $\pm \mathrm{SD}$ calculated from the log-transformed distribution

${ }^{\mathrm{c}}$ Note that glucose concentrations reported for CVD events vs no CVD events exclude those with diabetes

TIA, transient ischaemic attack

(Table 2). This observation held regardless of a history of vascular disease at baseline or not $(p>0.15)$. The same was also true in fully adjusted analyses.

Among 4,945 participants without baseline diabetes, 292 developed new-onset diabetes (248 on the basis of serial glucose measurements, and the remaining 44 on the basis of new prescriptions for oral diabetes medications). In keeping with expectations, predictors for new-onset diabetes were fasting glucose (HR 3.90, $p<0.001$ per $\mathrm{mmol} / 1$ increases), BMI (HR 1.09, $p<0.001$, per BMI unit) and triacylglycerol (HR 1.68, $p=0.0027$ per unit $\log [$ triacylglycerol]). For ICAM-1 the HR in treatment-adjusted analyses was 1.60 (95\% CI 1.14-2.25, $p=0.0065$ ), with little attenuation of the association upon adjustment for traditional ' $\mathrm{CVD}$ risk factors' (HR 1.82, 95\% CI 1.27-2.63, $p=0.0013$ ) (Table 2). Further adjustment for fasting glucose did not attenuate the association (HR 1.85, 95\% CI 1.27-2.70, $p=0.0012$ ), nor did additional adjustment for $\log [\mathrm{CRP}]$ (HR 1.84, 95\% CI $1.26-2.69, p=0.0015$ ).

Finally, when we examined associations using $1 \mathrm{SD}$ change in ICAM-1, the HR was 1.01 (95\% CI 0.94-1.08) for CVD events and 1.22 (95\% CI 1.08-1.39) for incident diabetes.

\section{Discussion}

This study in the elderly adds additional strong support for an authentic association of elevated ICAM-1 with risk of incident diabetes, despite no association with incident CVD. This is, as far as we are aware, the first study to test simultaneously the association of baseline ICAM-1 with both incident diabetes and CVD events. 
Table 2 HRs (95\% CIs) for a 1 unit increase in $\log [$ ICAM-1] vs risk of incident CVD events and incident diabetes

\begin{tabular}{|c|c|c|c|c|c|c|}
\hline \multirow[t]{2}{*}{ Endpoint } & \multicolumn{2}{|l|}{ Model A } & \multicolumn{2}{|l|}{ Model B } & \multicolumn{2}{|l|}{ Model C } \\
\hline & $\mathrm{HR}(95 \% \mathrm{CI})$ & $p$ value & $\mathrm{HR}(95 \% \mathrm{CI})$ & $p$ value & $\mathrm{HR}(95 \% \mathrm{CI})$ & $p$ value \\
\hline \multicolumn{7}{|l|}{ Incident CVD event ${ }^{\mathrm{a}}$} \\
\hline Entire cohort $(n=5,685) ; 866$ events & $1.13(0.93-1.38)$ & 0.23 & $1.02(0.83-1.27)^{\mathrm{b}}$ & 0.81 & $0.98(0.80-1.22)^{\mathrm{b}}$ & 0.89 \\
\hline No prior CVD $(n=3,168) ; 374$ events & $1.23(0.91-1.67)$ & 0.17 & $1.09(0.79-1.49)$ & 0.61 & $1.03(0.75-1.42)$ & 0.85 \\
\hline \multicolumn{7}{|l|}{ Incident diabetes } \\
\hline No baseline diabetes $(n=4,945) ; 292$ events & $1.60(1.14-2.25)$ & 0.0065 & $1.82(1.27-2.63)$ & 0.0013 & $1.84(1.26-2.69)^{\mathrm{c}}$ & 0.0015 \\
\hline
\end{tabular}

Note that the association of ICAM-1 with risk of CHD (CHD death or non-fatal myocardial infarction) or stroke (fatal or non-fatal stroke) as separate endpoints were similar to the results for total CVD events (data available on request)

Model A, adjusted for randomised treatment

Model B, adjusted for randomised treatment, age, sex, BMI, LDL-cholesterol, HDL-cholesterol, log[triacylglycerol], systolic BP, diastolic BP, current smoker, ex smoker, diabetes (in CVD outcomes only), use of antihypertensive therapy and country

Model $\mathrm{C}=$ Model $\mathrm{B}+\log [\mathrm{CRP}]$

${ }^{\mathrm{a}} \mathrm{CHD}$ death or non-fatal myocardial infarction or fatal or non-fatal stroke

${ }^{\mathrm{b}}$ Additionally adjusted for history of CHD, peripheral arterial disease, stroke or transient ischaemic attack

${ }^{\mathrm{c}}$ Model $\mathrm{C}+$ glucose

A meta-analysis has reported univariate, but not multivariate, associations of ICAM-1 with CHD [5]. In the present elderly population of men and women, even univariate associations with vascular events were absent, and this was true for participants with or without a history of vascular disease. This lack of association may reflect a general agerelated weakening of risk factor associations with vascular events. Accordingly, it is even more remarkable that high ICAM-1 was significantly associated with diabetes risk, even after adjustment for traditional risk factors and fasting glucose and CRP. These findings suggest ICAM-1 levels, in as much as they may reflect endothelial activation, are more strongly related to diabetes risk in the elderly than they are to cardiovascular risk. These data extend existing data in middle-aged individuals, since, in general, reported associations of circulating ICAM-1 with diabetes risk $[3,4]$ appear to be of greater magnitude than their associations with CVD risk [5].

Why ICAM-1 should better predict incident diabetes is unknown. Speculatively, since there is much more microvascular than large artery endothelium, one could assume that concentrations of ICAM-1 are determined more by microcirculatory function. This is relevant since endothelial perturbations in small vessels influence nutrient delivery and thus glucose metabolism. Alternatively, since ICAM-1 levels are not specific for endothelial activation, perturbances in other pathways may account for associations of elevated ICAM-1 with diabetes. The search for responsible mechanisms is important and may yield novel therapeutic mechanisms to treat or prevent diabetes.

Strengths and limitations The endpoint numbers in this study are large by most standards. The study was conducted in the context of a randomised trial of statins [9]; however, baseline measures were taken before randomisation, and there was no significant interaction by randomised treatment. The study had an average 3.2 years of follow-up, although short follow-up may be appropriate in the more elderly because of the high event risk, and others have shown no difference in the association of ICAM-1 with vascular events by longer follow-up [4]. We acknowledge that the lack of OGTTs means that some individuals may have developed type 2 diabetes not detectable by changes in fasting glucose alone. However, lack of OGTTs would mean missed cases of diabetes in controls, but assuming similar baseline elevations in ICAM-1 in this latter group, then this would have biased the study against a link between ICAM-1 and incident diabetes. Moreover, the strongest predictors for incident diabetes in this population (fasting glucose, BMI and triacylglycerol) concur with expectations. Finally, whilst we accept that since many risk variables lessen in their association with vascular events in the elderly, most events, both diabetes and CVD events, occur increasingly in these populations, so the results are of interest.

\section{Conclusion}

We have shown for the first time that elevated ICAM-1 levels are associated with risk of diabetes but not CVD events (CHD or stroke combined or separately) in the elderly. We propose that endothelial dysfunction, at least as 'estimated' by elevated circulating ICAM-1 levels may be aligned more to diabetes than to vascular risk. The aetiological mechanisms underpinning these data require future investigation. 
Acknowledgements We thank L. Cherry for conducting the ICAM-1 assays. We thank the Stroke Association, UK, for funding the ICAM-1 analyses. The organisation played no part in the design, analysis, interpretation or reporting of the data.

Duality of interest The authors declare that there is no duality of interest associated with this manuscript.

\section{References}

1. Hwang SJ, Ballantyne CM, Sharrett AR et al (1997) Circulating adhesion molecules VCAM-1, ICAM-1, and E-selectin in carotid atherosclerosis and incident coronary heart disease cases: the Atherosclerosis Risk In Communities (ARIC) study. Circulation 96:4219-4225

2. Ridker PM, Hennekens CH, Buring JE, Rifai N (2000) C-reactive protein and other markers of inflammation in the prediction of cardiovascular disease in women. N Engl J Med 342:836-843

3. Ridker PM, Hennekens CH, Roitman-Johnson B, Stampfer MJ, Allen J (1998) Plasma concentration of soluble intercellular adhesion molecule 1 and risks of future myocardial infarction in apparently healthy men. Lancet 351:88-92

4. Tanne D, Haim M, Boyko V et al (2002) Soluble intercellular adhesion molecule-1 and risk of future ischemic stroke: a nested case-control study from the Bezafibrate Infarction Prevention (BIP) study cohort. Stroke 33:2182-2186

5. Malik I, Danesh J, Whincup P et al (2001) Soluble adhesion molecules and prediction of coronary heart disease: a prospective study and meta-analysis. Lancet 358:971-976

6. Meigs JB, Hu FB, Rifai N, Manson JE (2004) Biomarkers of endothelial dysfunction and risk of type 2 diabetes mellitus. JAMA 291:1978-1986

7. Song Y, Manson JE, Tinker L et al (2007) Circulating levels of endothelial adhesion molecules and risk of diabetes in an ethnically diverse cohort of women. Diabetes 56:1898-1904

8. Sattar N, Wannamethee SG, Forouhi NG (2008) Novel biochemical risk factors for type 2 diabetes: pathogenic insights or prediction possibilities. Diabetologia 51:926-940

9. Sattar N, McConnachie A, Shaper AG et al (2008) Can metabolic syndrome usefully predict cardiovascular disease and diabetes? Outcome data from two prospective studies. Lancet $371: 1927-1935$ 